\section{Ankylography Permits Three-Dimensional Structure Determination from a Single Sample Orientation}

Traditionally, three-dimensional (3D) imaging using coherent diffraction microscopy requires multiple diffraction patterns at different sample orientations. To generate these patterns, precise mechanical tilting or multiple identical copies of a specimen are necessary, thereby preventing 3D imaging by a single $x$-ray free electron laser (X-FEL) pulse or time-resolved 3D structure determination of disordered materials. J. Miao and K.S. Raines of the University of California, Los Angeles (UCLA), H.C. Kapteyn at the University of Colorado, Boulder, and J. Du at the University of North Texas, and co-researchers, however, have developed a novel imaging technique termed ankylography (from the Greek ankylos for "curved" and graphein for "writing"), which under certain conditions can reconstruct 3D structural information from a single exposure using a monochromatic incident beam. While requiring a similar amount of incident flux to achieve a desired resolution as compared to conventional 3D coherent diffraction microscopy, their approach eliminates the necessity of sample tilting by redistributing the intensities more finely on the Ewald sphere. The researchers state that, with further development, this 3D structure determination method could find broad applications in the physical and life sciences.

As described in the January 14 issue of Nature (DOI: 10.1038 /nature08705; p. 214), the conceptual design of ankylography begins with a coherent beam illuminating a finite object and the scattered waves forming a diffraction pattern on the Ewald sphere. The researchers demonstrate that when the 2D spherical diffraction pattern is sampled at a sufficiently fine scale, the oversampled diffraction pattern can, in theory, be used to determine the 3D structure of the object. A phase retrieval algorithm that iterates back and forth between Fourier and real space is used to compute the 3D reconstruction. In their studies, the constraints of non-negativity, uniformity outside the support, continuity inside the support, and amplitude extension were applied to facilitate these reconstructions. To confirm the theoretical analysis, 3D numerical reconstructions of a sodium silicate glass structure at $2 \AA$ resolution and a single poliovirus at $2-3 \mathrm{~nm}$ resolution were computed using only 2D spherical diffraction patterns. The researchers further demonstrated the experimental feasibility of ankylography by obtaining a 3D image of a test object from a single $2 \mathrm{D}$ diffraction pattern taken with a soft $\mathrm{x}$-ray laser.

Because of the oversampling requirement of the single $2 \mathrm{D}$ diffraction pattern, in order to compute 3D reconstructions of large specimens at high resolutions, ankylography requires area detectors with a large number of pixels. Additionally, spherical area detectors are more advantageous in reducing interpolation errors. "By incorporating additional constraints into the phase retrieval algorithm and using dedicated detectors," the researchers said that "ankylography could be applied to perform 3D structure determination of a broad range of specimens without the necessity of sample tilting, scanning, or sectioning."

TIFFANY D. ZIEBELL

\section{Electronic Liquid Crystal States Discovered in Parent of Iron-Based Superconductor}

An international team of researchers has discovered evidence for electronic liquid crystal states within the parent compound of one type of iron-based, high-temperature (high- $T_{\mathrm{c}}$ ) superconductor.

"Because these findings appear similar to what we have observed in the parent state of cuprate superconductors, it suggests this could represent a common factor in the mechanism for high- $T_{\mathrm{c}}$ superconductivity in these two otherwise very different families



Nanostructure meets Calorimetry

\section{S3-MICROcaliX}

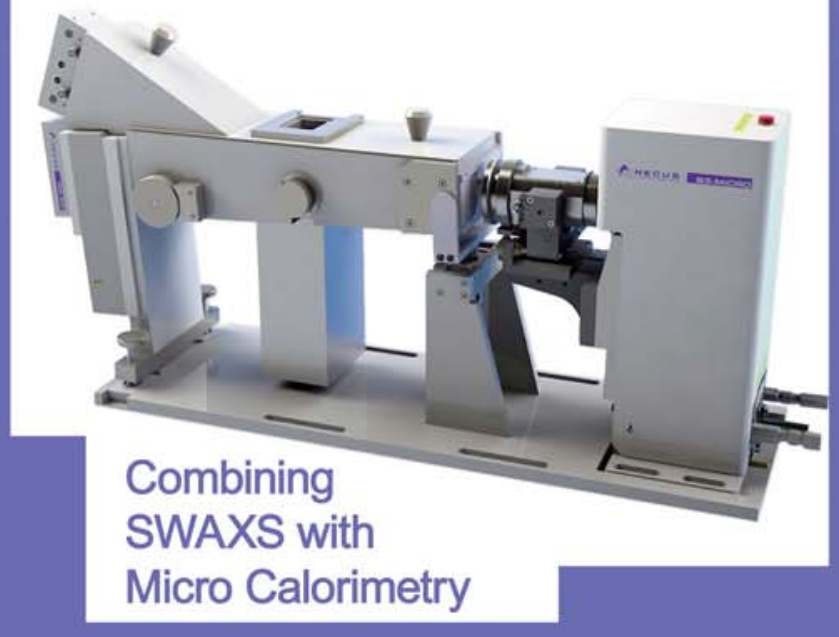

Absolute SAXS

Zero Background

Highest SAXS Resolution

\section{Unique SWAXS Instrument \\ Separate, parallel-readout \\ Position Sensitive Detectors}

Uniquely embedded CALORIMETER

Sensitivities fully matched

Direct access to sample

Unique calorimetric design

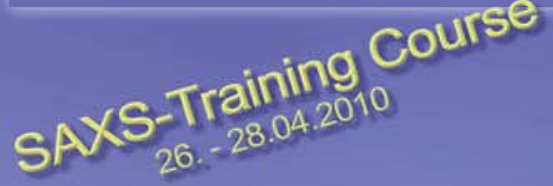

www.hecus.at 